\title{
Eu anomalies in lunar plagioclase reflect secondary processing by subsolidus reequilibration and introduction of a KREEP component
}

\author{
DIAN JI AND NICK DYGERT
}

University of Tennessee, Knoxville

Presenting Author: jd01811@163.com

The anorthositic lunar crust, predominantly consisting of anorthites characterized by significant positive $\mathrm{Eu}$ anomalies, is an important product of lunar magma ocean (LMO) solidification. We calculated REE and Eu distributions in lunar anorthosites according to recent experimentally-determined fractional crystallization experiments [e.g., 1,2]. Applying the plagioclase-melt Eu and REE partitioning models of [3] and [4], and making lunar-relevant $f \mathrm{O}_{2}$ and LMO bulk composition assumptions, we find that crystalized plagioclase exhibit lager $\mathrm{Eu}$ anomalies and flatter REE patterns (i.e., lower $\mathrm{Ce} / \mathrm{Sm}$ ) than Apollo samples [5]. In this study, we explore possible causes of this phenomenon.

We first considered remelting of the anorthosites. We calculated REE abundances of solid in equilibrium with instantaneous fractional melts. Although the recrystallized plagioclases show an elevated $\mathrm{Ce} / \mathrm{Sm}$, these scenarios exacerbate the Eu anomaly discrepancy. We explored $T$ - and $f \mathrm{O}_{2}$ dependent closed-system subsolidus reequilibration, which expands the space of $\mathrm{Ce} / \mathrm{Sm}$ as well as $\mathrm{Eu}$ anomalies, however, the variation of $\mathrm{Eu}$ anomalies is still limited and cannot fit many natural samples under $f \mathrm{O}_{2}$ conditions relevant to a reduced Moon. We then tested subsolidus reequilibration after addition of a chondritic component, which also failed to reproduce the natural distributions. Finally, we explored subsolidus reequilibration after addition of a KREEP component.

Addition of minor KREEP reduces Eu anomalies and elevates $\mathrm{Ce} / \mathrm{Sm}$ ratios, reproducing variations in the natural samples. The proportion of KREEP required depends on the bulk LMO composition assumed. Addition of a KREEP component implies secondary magmatic processing of the lunar anorthosites, perhaps associated with Serial or Mg-suite Magmatism after a cumulate mantle overturn event.

[1] Rapp \& Draper (2018) MAPS 53, 1452-1455. [2] Charlier et al (2018) GCA 234, 50-69. [3] Dygert et al (2020) GCA 279, 258-280. [4] Sun et al (2017) GCA 206, 273-295 [5] PernetFisher et al (2019) GCA 266, 109-130. 